\title{
Radiologie - Das Drehkreuz in der Medizin: Hygiene in der Radiologie
}

\author{
Radiology - A Major Hub in Medicine
}

A. Saur

Kaum ein Patient hat heutzutage keinen Berührungspunkt mit einem der vielen bildgebenden Verfahren der Radiologie. Denn fast alle klinischen Entscheidungen, wie z. B. Klärung der statio-

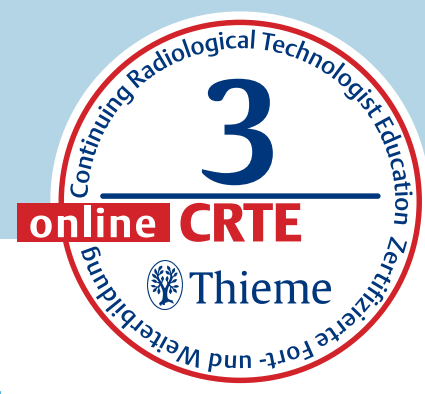
nären Aufnahme bzw. Entlassung, Indikation für eine Operation, Therapieeinleitung oder Therapieverlauf, werden mittels einer oder mehrerer der vielseitigen radiologischen Verfahren getroffen. Die Radiologie hat sich als Drehkreuz in der Medizin etabliert.

Durch diese Tatsache und aufgrund kürzerer Untersuchungszeiten - bedingt durch die rasante technische Weiterentwicklung der Geräte - haben sich im Laufe der Zeit das Patientenaufkommen bzw. der Patientendurchlauf in einer radiologischen Abteilung stark erhöht. Dies hat zur Folge, dass Mitarbeiter der Radiologie, insbesondere MTRA, viel häufiger den direkten Kontakt zu sowohl ambulanten als auch stationären Patienten haben. Daher gewinnt auch im Rahmen der (Krankenhaus-)Hygiene die Radiologie immer mehr an Bedeutung. Das Risiko einer Verschleppung von Infektionen mit multiresistenten Erregern muss minimiert werden, um - neben dem Risiko der Mortalität und Morbidität für Patienten ebenso wie für das Personal - das Kostenrisiko durch notwendige Ausweitung der Therapiemaßnahmen und Verlängerung von Liegezeiten so gering wie möglich zu halten. Hierzu können an den Workflow einer radiologischen Abteilung angepasste Hygienemaßnahmen einen wichtigen Beitrag leisten, wenn diese durch das Personal konsequent umgesetzt werden. Die Radiologie als ein Drehkreuz in der Medizin und der Hygiene!

Das Risiko einer Verschleppung von Infektionen mit multiresistenten Erregern muss minimiert werden, um das Kostenrisiko durch notwendige Ausweitung der Therapiemaßnahmen und Verlängerung von Liegezeiten so gering wie möglich zu halten.

\section{Einleitung}

Nosokomiale Infektionen durch multiresistente Erreger (MRSA, MRGN, ESBL) stellen aufgrund eingeschränkter Therapiemöglichkeiten ein besonderes Risiko für infizierte Patienten dar. Derzeit geht man schätzungsweise - allein durch MRSA verursacht - europaweit von jährlich ca. 170000 Infektionen und über 5000 Todesfällen aus [8]. Infolgedessen belastet dies die europäischen Gesundheitssysteme bzw. Kostenträger aufgrund der notwendigen Therapieausweitung mit mehr als 1 Mio. zusätzlichen Krankenhausaufenthaltstagen und damit verbundenen Kosten von ca. 380 Mio. € pro Jahr [8].

Dies führte im August 2011 zu Änderungen des Infektionsschutzgesetzes (IfSG) und hat maßgeblich die fachübergreifende Umsetzung vorbeugender hygienischer Maßnahmen für den Krankenhausbetrieb beeinflusst [6]. In der aktuellen Version des IfSG werden insbesondere notwendige infrastrukturelle Anforderungen konkretisiert, wobei die genauen Anforderungen durch die jeweilige Länderverordnungen in der sog. Verordnung über die Hygiene und Infektionsprävention in medizinischen Einrichtungen (HygMedVO) geregelt werden [7]. Hier werden u.a. neben den hygienebeauftragten Ärzten ( $\$ 1$ Abs.1) auch weitere nicht näher definierte Hygienebeauftragte ( $§ 1$ Abs.2) genannt. Diese sollen „mindestens sicherstellen, dass innerbetriebliche Verfahrensweisen zur Infektionshygiene in Hygieneplänen festgelegt sind“. Folglich ist es sinnvoll, neben dem Hygienebeauftragten (Radiologen) auch MTRA zu Hygienebeauftragten zu benennen, damit sie im Team in Zusammenarbeit mit dem Krankenhaushygieniker an eine radiologische $\mathrm{Ab}$ teilung angepasste fachgerechte Hygienemaß- 
nahmen erstellen, die den Workflow möglichst wenig unterbrechen oder verlangsamen.

Hygienebeauftragte sind verpflichtet, sich fortzubilden und bedürfen dementsprechend der regelmäßigen Fortbildung in längstens 2-jährigem Abstand [7]. Dieser Artikel soll einen Überblick zu allgemeinen Infektionspräventionsmaßnahmen sowie Empfehlungen zu ihrer praktischen Durchführung geben. Dabei werden erregerspezifische Hygienemaßnahmen vorgestellt, um die z.T. kosten- und/oder zeitintensiven Verfahrensweisen gezielt und damit im notwendigen Umfang einsetzen zu können.

Für Hygienebeauftragte ist die Fortbildung in längstens 2-jährigem Abstand Pflicht.

\section{Aufgaben hygienebeauftragte Person [5]}

- Mitwirkung bei der Erstellung von Hygieneplänen gem. §36 IfSG

> regelmäßiges Überwachen der Einhaltung von Regeln bezüglich der Hygiene- und Infektionsprävention

- Verbesserung der Hygienepläne und deren Funktionsabläufe

- Mitwirkung bei der Verhütung und Bekämpfung von nosokomialen Infektionen durch allgemeine und bereichsspezifische Beratung bei regelmäßiger Begehung aller Bereiche einer Abteilung

- regelmäßige Überwachung von Arbeitsabläufen, wie z.B.:

> Desinfektionsmaßnahmen

$>$ Reinigung

Entsorgung

- Schulung der Mitarbeiter in Bezug auf Krankenhaushygiene einschließlich:

- Hinweise auf Gesetze

V Verordnungen

$>$ Richtlinien

> anerkannte Regeln der Technik

- Mitwirkung bei der Auswahl von Produkten, z.B.:

Desinfektionsmittel

$\checkmark$ Medizinprodukte

- ggf. Mitwirkung bei der Planung funktioneller und baulicher Maßnahmen
Hintergrundwissen:

Nosokomiale Infektion [10]

Nosokomiale Infektion oder auch Krankenhausinfektion bedeutet, dass die Infektion während eines stationären oder ambulanten Krankenhausaufenthalts erworben wurde. Gleiches gilt auch für den Aufenthalt in Pflegeeinrichtungen. Dabei dürfen keine Hinweise einer bereits vorhandenen oder aber bereits in der Inkubationsphase befindlichen Infektion existieren.

Die häufigsten Krankenhausinfektionen sind:

- Harnwegsinfekt

- Sepsis

Pneumonie (Beatmungspneumonie)

- Wundinfektion

Die Rate von nosokomialen Infektionen steht

im Zusammenhang folgender Faktoren:

- Alter der Patienten

- Häufigkeit und Schwere von Begleiterkrankungen

- Einsatz operativer Techniken

- Therapien, die die Immunabwehr herabsetzen

- Wissensstand des Personals zum Thema Hygiene

\section{Grundlagen der Hygiene in der Radiologie - Allgemeine hygienische Maßnahmen}

\section{Händedesinfektion}

Hierbei unterscheidet man 2 Arten zur Händedesinfektion. Zum einen gibt es eine chirurgische, zum anderen eine hygienische Händedesinfektion. Letztere dient in erster Linie der Beseitigung der transienten Hautflora und beinhaltet in der Regel das einmalige Verreiben dementsprechend geeigneter Präparate (z.B. Desderman pure, Skinsept F, Spitacid, Sterillium) in den trockenen Händen über einen Zeitraum von mindestens ca. 30s (Einwirkzeit). Dabei sollte man darauf achten, die Hautoberfläche vollständig zu benetzen ( $\bullet$ Abb.1). Da die menschliche Haut sehr empfindlich ist, sollten alkoholische Präparate verwendet werden. Es gilt zu beachten, dass diese nach Anbruch max. 6 Monate haltbar sind.

Für die hygienische Händedesinfektion darf die Länge der Fingernägel die Fingerkuppen nicht überschreiten. Nagellack, künstliche Fingernägel, Ringe und Armbanduhr sind nicht erlaubt. 

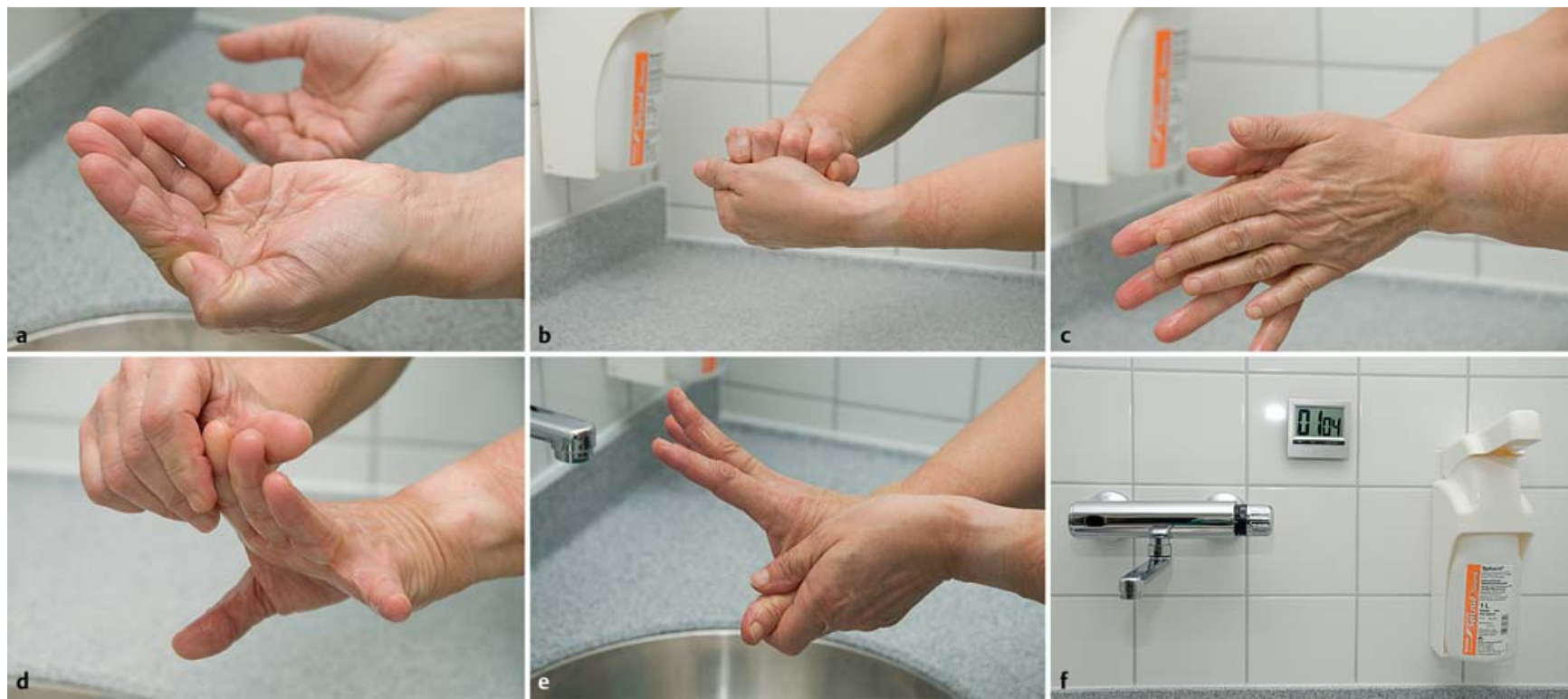

Abb.1 Durchführung der Händedesinfektion. Prinzipiell immer zu den Fingerspitzen ausstreichen.

a Einen Hub Desinfektionsmittel in die Hand.

b Desinfektion der Fingerkuppen. Schritt a und b wiederholen.

c Desinfektion der Handinnenflächen einschließlich Fingerzwischenräume.

d Desinfektion des Handrückens einschließlich Fingerzwischenräume.

e Desinfektion beider Daumen.

f Zeitaufwand ca. 60 Sekunden.

\section{Beispielhafter Ablauf einer Hände-}

desinfektion in radiologischen Instituten

Händedesinfektion ( $\bullet$ Abb.1) beim Anlegen von:

- Handschuhen

- Schutzkittel

- Mundschutz

- Haarschutz

- ggf. Spritzschutzbrille

Händedesinfektion nach dem Ablegen von:

- Handschuhen

- Schutzkittel

- Spritzschutzbrille, falls in Gebrauch

- Mund-Nasen-Schutz

- Haarschutz

Um Eigenübertragung durch kontaminierte Schutzkleidung zu vermeiden, ist diese bereits im Untersuchungsraum in entsprechender Reihenfolge abzulegen und als Kontaminationsmüll, d.h. separat von normalem Krankenhausmüll in entsprechenden Behältnissen, zu entsorgen.

\section{Zeitpunkt der Händedesinfektion}

- vor und nach jedem Patientenkontakt, auch wenn Schutzhandschuhe getragen wurden

- vor und nach invasiven Untersuchungen, auch wenn Handschuhe getragen wurden, z.B.:

$\rightarrow$ Angiografie

- Injektionen (Kontrastmittel)

$>$ Punktionen

- Legen bzw. Entfernen von Venenverweilkanülen

- vor Tätigkeiten mit Kontaminationsgefahr für den Patienten, z.B.:

- Aufziehen von Medikamenten

- Bereitstellen von Infusionen

- Herstellen von Mischinfusionen

- nach Kontakt mit potenziell kontaminierten Gegenständen, Flüssigkeiten oder Flächen,

z.B.:

Schmutzwäsche

$>$ Abfälle

- vor Anlegen von Schutzkleidung sowie nach deren Ablegen:

Handschuhe

> Schutzkittel

> Mundschutz

Haarschutz

- nach Husten, Niesen und Naseputzen

- vor Essenszubereitung bzw. Nahrungsaufnahme

- bei Bedarf 


\section{Flächendesinfektion \\ $\nabla$}

Neben der Händedesinfektion bildet die Flächendesinfektion mit dementsprechend zugelassenen Flächendesinfektionsmitteln nach VHAl-Liste [9] die 2. wichtige Säule in der Hygieneprävention nosokomialer Infektionen. Oberflächen und Gerätschaften sollten in festgelegten Zeitabständen sowie nach jedem Patientenkontakt desinfiziert werden ( $\bullet$ Tab. 1). Somit kann die Bildung von Erregerreservoiren ( $\bullet$ Abb.2-5) über einen längeren Zeitraum verhindert werden. Daneben sollte täglich durch geschultes Reinigungspersonal unabhängig von o.g. Oberflächendesinfektion eine Raumreinigung und -desinfektion, ggf. mehrmals täglich, durchgeführt werden.

Um den Zeitaufwand der Wischdesinfektion nach jedem Patienten - insbesondere nach infektiösen - zu reduzieren, ist es sinnvoll, Oberflächen, mit denen der Patient in Kontakt kommt, mit diversen Einmalartikeln als Hilfsmittel abzudecken ( Tab.2), die relativ schnell und einfach nach jedem Patienten gewechselt werden können. Ebenso sollten Materialien, die im Untersuchungsraum gelagert sind, in geschlossenen Schränken bzw. Behältnissen verwahrt werden. Prinzipiell sollten sich so wenig Materialien und bewegliche Gegenstände wie möglich im Untersuchungsraum befinden.

Tab. 1 Desinfektion von Geräteoberflächen.

\begin{tabular}{|c|c|c|c|c|}
\hline Röntgen & $C T$ & MRT & $\begin{array}{l}\text { Angiografie/ } \\
\text { Durchleuchtung }\end{array}$ & $\begin{array}{l}\text { Ultra- } \\
\text { schall }\end{array}$ \\
\hline $\begin{array}{l}\text { Griffe der } \\
\text { Röntgenröhre } \\
\text { Kassetten- }\end{array}$ & Dantry & $\begin{array}{l}\text { Kopfhörer } \\
\text { Notfallklingel }\end{array}$ & $\begin{array}{l}\text { Bildverstärker } \\
\text { Instrumenten- } \\
\text { tisch }\end{array}$ & \multirow[t]{2}{*}{$\begin{array}{l}\text { Schall- } \\
\text { köpfe }\end{array}$} \\
\hline $\begin{aligned} & \text { schublade } \\
& \text { Rasterwandstativ } \\
& \text { Tiefenblende } \\
& \text { Kassetten/ } \\
& \text { Detektoren }\end{aligned}$ & \multicolumn{3}{|c|}{ Kontrastmittelinjektionsautomaten } & \\
\hline $\begin{aligned} & \text { Untersuchungstisc } \\
& \text { Bedienknöpfe der } \\
& \text { Patientenlagerung } \\
& \text { Patientenumlager } \\
& \text { Bleischürzen } \\
& \text { Gonadenschutz } \\
& \text { PC-Tastatur/-Maus } \\
& \text { Telefonhörer/-tast } \\
& \text { Türklinken } \\
& \text { Schrank-/Schublad } \\
& \text { Lichtschalter } \\
& \text { Klemmbrett für Au }\end{aligned}$ & $\begin{array}{l}\text { iege } \\
\text { ate } \\
\text { fen, wischf } \\
\text { shilfen (z. }\end{array}$ & $\begin{array}{l}\text { (z. B. Kopfkissen } \\
\text { Rollboard) }\end{array}$ & kenrolle, diverse $\mathrm{K}$ & \\
\hline
\end{tabular}

Die Aufbereitung eines Arbeitsplatzes vor Arbeitsbeginn und am Ende eines Arbeitstags sollte zu den ersten grundlegenden Hygienemaßnahmen gehören. Prinzipiell müssen bei jeder Flächen- und Gerätedesinfektion die Oberflächen vollständig benetzt sein; ebenso ist das Abtrocknen abzuwarten (Einwirkzeit).

Oberflächen und Gerätschaften sollten in festgelegten Zeitabständen sowie nach jedem Patientenkontakt desinfiziert werden.

\section{Kleiderordnung}

Berufskleidung wird vom Arbeitgeber gestellt ( $\bullet$ Tab.3). Ebenso wird die Reinigung dieser Kleidung durch den Arbeitgeber organisiert. Die Berufskleidung sollte nicht zu Hause gewaschen werden. Das Tragen von Privatkleidung über Berufs- oder Bereichskleidung ist zu vermeiden.

Es gilt zu beachten, dass unsterile Handschuhe mikroskopisch kleine Löcher aufweisen können. Somit kann ein 100\%-iger Schutz vor Mikroorganismen nicht gewährleistet werden. Gleiches gilt ebenfalls für sterile Handschuhe. Auch hier können Defekte, wenn auch im geringeren Maße, nicht ausgeschlossen werden [4]. Daraus ergibt sich die prinzipielle Händedesinfektion vor und nach dem Tragen von Schutzhandschuhen (๑ Abb.6).

Wie für alle Bereiche eines Krankenhauses muss es auch für radiologische Abteilungen einen entsprechenden Hygiene- und Desinfektionsplan u. a. in Form von Maßnahmenblättern für unterschiedliche Infektionskrankheiten geben, in dem verbindlich der allgemeine Rahmen bezüglich der Hände- und Flächendesinfektion sowie die Kleiderordnung vorgegeben wird.

Ein Hygiene- und Desinfektionsplan muss in jeder radiologischen Abteilung vorhanden sein. Dessen Vorgaben sind verbindlich. 


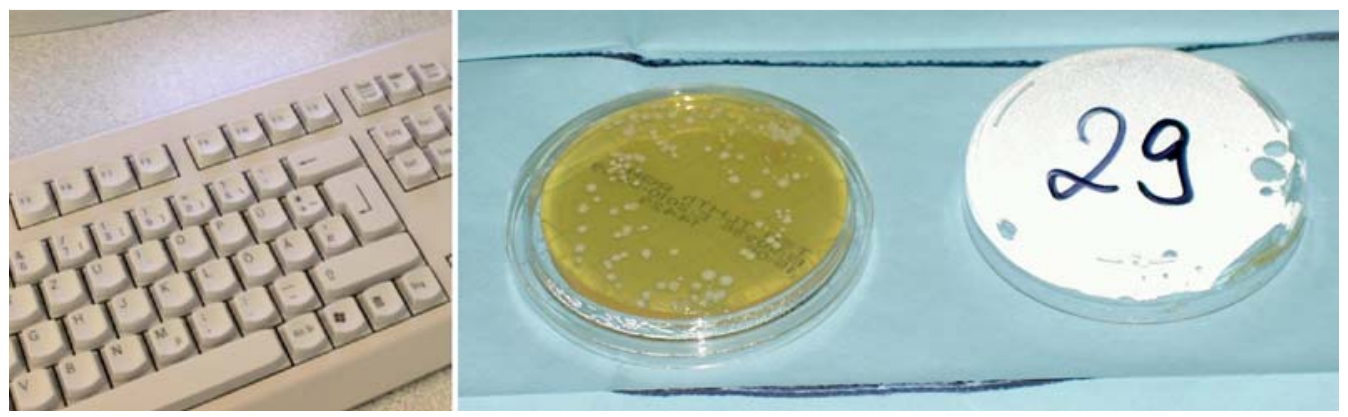

Abb. 2 Abklatschprobe Tastatur.
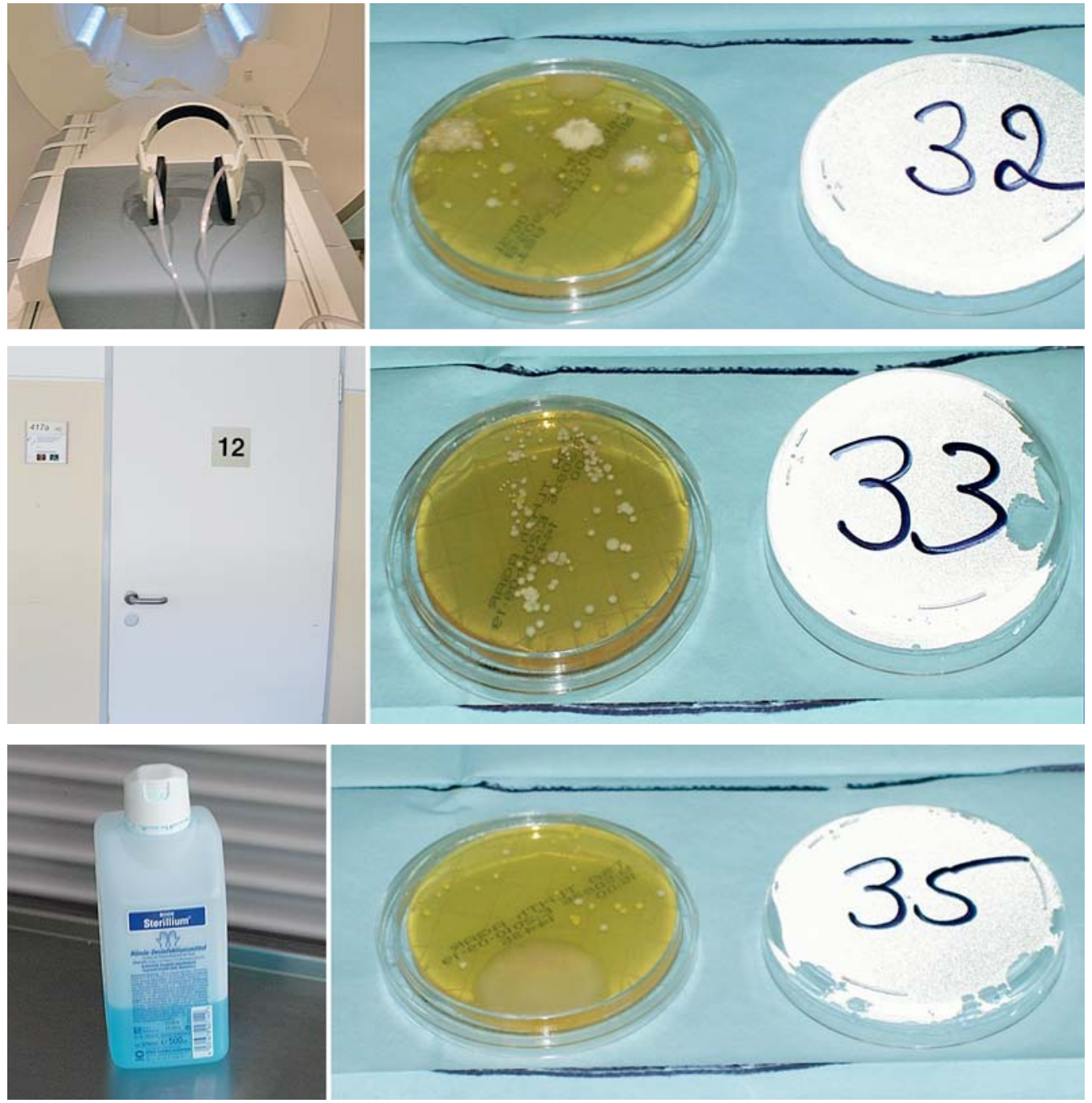

Abb. 3 Abklatschprobe MRT-Kopfhörer.

Abb.4 Abklatschprobe Patientenkabine Türgriff.

Abb. 5 Abklatschprobe Handflasche.

Tab.2 Abdeckungsmöglichkeiten von Geräteoberflächen.

\begin{tabular}{|c|c|c|c|c|}
\hline Röntgen & Ст & MRT & Angiografie/Durchleuchtung & Ultraschall \\
\hline $\begin{array}{l}\text { Tiefenblende: Bildver- } \\
\text { stärkerhaube } \\
\text { Kassetten/Detektoren: } \\
\text { Einmaltüten } \\
\text { Rasterwandstativ: Krepp } \\
\text { oder Folie }\end{array}$ & & $\begin{array}{l}\text { Kopfhörer: } \\
\text { Überzieher } \\
\text { Notfallklingel: } \\
\text { Einmaltüten }\end{array}$ & $\begin{array}{l}\text { Bildverstärker: Bildverstär- } \\
\text { kerhaube } \\
\text { Instrumententisch: sterile } \\
\text { Operationsabdecktücher } \\
\text { mit gummierter Unterseite } \\
\text { Bedienknöpfe: Bildverstär- } \\
\text { kerhaube }\end{array}$ & $\begin{array}{l}\text { Bedienknöpfe: } \\
\text { Bildverstärker- } \\
\text { haube }\end{array}$ \\
\hline \multicolumn{5}{|c|}{$\begin{array}{l}\text { Untersuchungstisch/-liege: Stecklaken, Krepppapier; bei Verdacht auf bzw. bekannter Infektion: Bettfolien } \\
\text { Patientenlagerungshilfen (z. B. Kopfkissen, Nackenrolle, diverse Keile): Tüten }\end{array}$} \\
\hline
\end{tabular}


Tab. 3 Kleiderordnung.

\begin{tabular}{|c|c|c|c|}
\hline Bezeichnung & Definition & Tragen der Kleidung & Wechsel der Kleidung \\
\hline Berufskleidung & Grundschutzfunktion & $\begin{array}{l}\text { während der Arbeitszeit - vorne } \\
\text { geschlossen }\end{array}$ & $\begin{array}{l}\text { mind. } 2 \text { x wöchentlich } \\
\text { sofort bei optisch sichtbarer Ver- } \\
\text { schmutzung oder Kontamination }\end{array}$ \\
\hline Bereichskleidung & $\begin{array}{l}\text { Ersatz der Berufsbekleidung in } \\
\text { Bereichen mit besonderer Infek- } \\
\text { tionsgefährdung, z. B.: } \\
\text { Angiografie } \\
\text { - Operationssaal } \\
\text { Intensivstation setzt sich farblich } \\
\text { von der Berufsbekleidung ab }\end{array}$ & & $\begin{array}{l}\text { täglich } \\
\text { sofort bei optisch sichtbarer Ver- } \\
\text { schmutzung oder Kontamination } \\
\text { bei Verlassen des Bereichs oder } \\
\text { Überzug eines Laufkittels } \\
\text { vor An- bzw. Ablegen der Bereichs- } \\
\text { kleidung: } \\
\text { hygienische Händedesinfektion! }\end{array}$ \\
\hline Laufkittel & Schutzkleidung & $\begin{array}{l}\text { bei Verlassen eines Bereiches mit } \\
\text { besonderer Infektionsgefährdung }\end{array}$ & \\
\hline Schutzkleidung & $\begin{array}{l}\text { in Biostoffverordnung (BioStoffVO): } \\
\text { persönliche Schutzausrüstung = } \\
\text { Personal- und Patientenschutz } \\
\text { zur Verhinderung von Keimver- } \\
\text { schleppung }\end{array}$ & $\begin{array}{l}\text { patienten- und aufgabenbezogen, } \\
\text { d. h. bei Gefahr der mikrobiellen } \\
\text { Kontamination und Keimverschlep- } \\
\text { pung }\end{array}$ & \\
\hline Schuhe & & $\begin{array}{l}\text { zur Vermeidung von Verletzungen } \\
\text { ausschließlich zweckmäßiges Schuh- } \\
\text { werk zulässig: } \\
\text { p geschlossen oder mit Fersenriemen } \\
\text { flacher Absatz oder durchgehende, } \\
\text { antistatische Gummisohle } \\
\text { in Bereichen mit besonderer Infek- } \\
\text { tionsgefährdung maschinell des- } \\
\text { infizierbar } \\
\text { vor An- bzw. Ablegen: } \\
\text { hygienische Händedesinfektion }\end{array}$ & \\
\hline $\begin{array}{l}\text { 1) Operationsmaske } \\
\text { (Mund-Nasen-Schutz) } \\
\text { 2) Partikelmaske, z.B. FFP2 }\end{array}$ & $\begin{array}{l}\text { Operationsmaske (Mund-Nasen- } \\
\text { Schutz) } \\
\text { zu 2): } \\
\text { für Personal mit Exspirationsventil } \\
\text { für Patienten ohne Exspirations- } \\
\text { ventil }\end{array}$ & $\begin{array}{l}\text { in Abhängigkeit von der jeweiligen } \\
\text { Infektionskrankheit 1) oder 2) } \\
\text { Mund, Nase, Barthaare sind } \\
\text { vollständig zu bedecken } \\
\text { darf nicht vorübergehend } \\
\text { abgenommen werden }\end{array}$ & $\begin{array}{l}\text { bei Durchfeuchten, } d . h \text {. wenn länger } \\
\text { als } 2-3 \mathrm{~h} \text { getragen }\end{array}$ \\
\hline Einmalhaube & Haarschutz & $\begin{array}{l}\text { in Abhängigkeit von der jeweiligen } \\
\text { Infektionskrankheit } \\
\text { in bestimmten Bereichen, z.B. } \\
\text { Angiografie } \\
\text { verdeckt die Haare vollständig }\end{array}$ & regelmäßig \\
\hline Schutzbrille & & $\begin{array}{l}\text { bei Gefahr von Verspritzen von Blut } \\
\text { oder Sekreten }\end{array}$ & \\
\hline
\end{tabular}

\section{Hygienische Aspekte bei der Applikation von Arzneimitteln}

\section{Manuell}

Vor dem ersten Anstechen von Behältnissen (z. B. Kontrastmittelflaschen, Infusionsflaschen) sollte eine Händedesinfektion durchgeführt und Handschuhe getragen werden. Da zeitlich versetzte Mehrfachentnahmen aus Behältnissen (sog. Multidosing) und deren Wiederbefüllung durch Aufsichtsbehörden wie das Bundesinstitut für Arzneimittel und Medizinprodukte (BfArM) nicht erlaubt sind, sollte man auf Einmaldosisbehälter mit minimalem Inhalt zurückgreifen. Denn die Gefahr der mikrobiellen Kontamination durch Mehrfachentnahmen über z.T. mehrere Tage aus ein und demselben Behältnis, das offen im Raum bei Raumtemperatur gelagert wurde, ist besonders hoch.
Die strikten Vorgaben des Arzneimittelgesetzes sind in den jeweiligen Gebrauchsinformationen der Hersteller hinterlegt und bei Verwendung von Arzneimitteln wie z. B. Kontrastmittel zwingend zu beachten.

\section{Maschinell}

$\nabla$

Zur i.v. Kontrastmittelapplikation werden neben der manuellen Einbringung semiautomatische Doppelspritzeninjektoren mit 2 parallel angeordneten Kolbenpumpen eingesetzt. D.h, diese Kolben sind über einen T-Schlauch und den sog. Patienteninjektionsschlauch mit der Venenverweilkanüle verbunden. Dies hat sich als Standard in der Schnittbilddiagnostik etabliert [5]. Hierbei erfolgt der Aufbau und die Befüllung der Kolben manuell, während die Applikation automatisch funktioniert. Auch hierbei gilt es den Hygiene- 


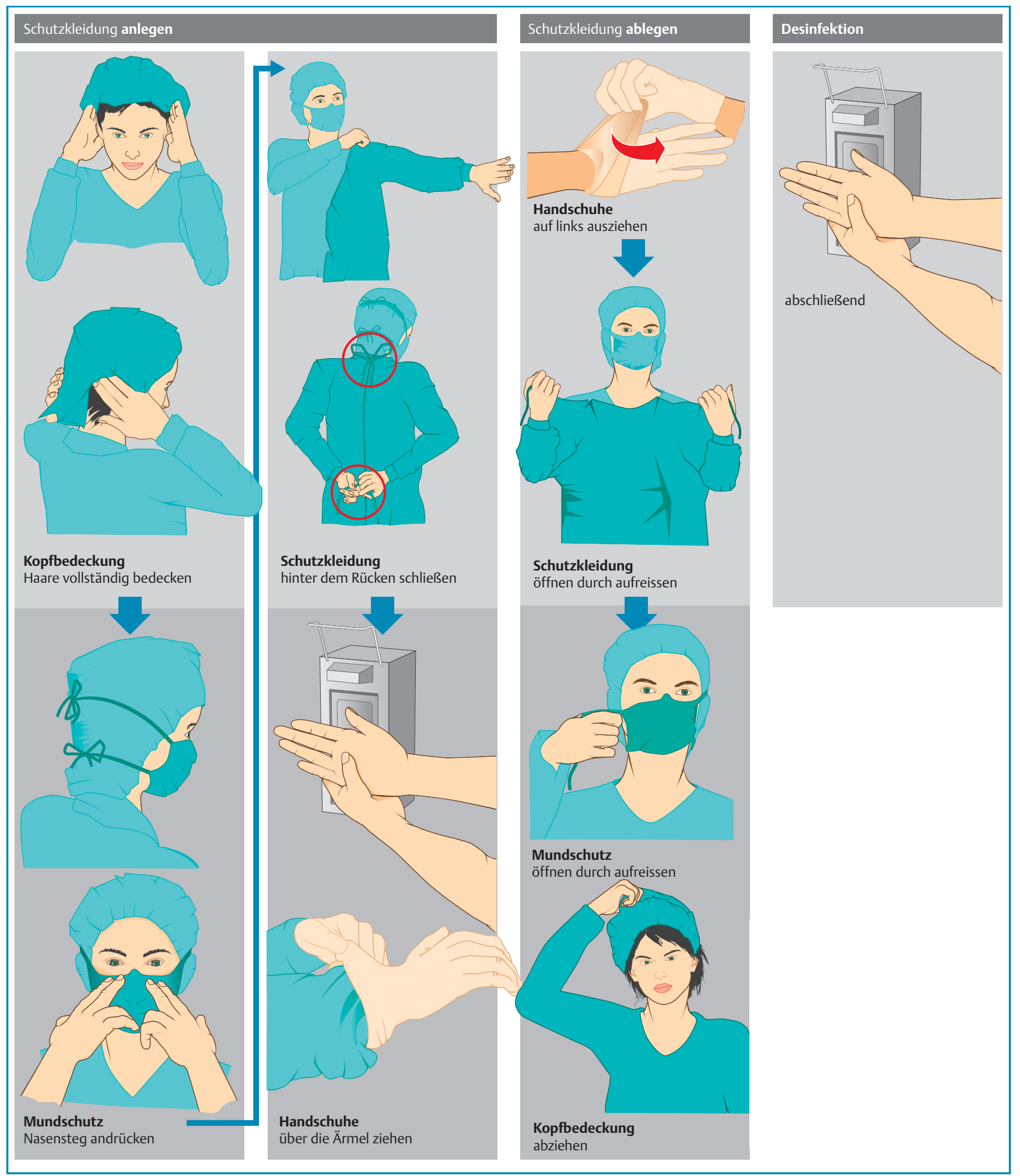

Abb. 6 Das korrekte An- und Ablegen von Schutzkleidung. 
standard - Händedesinfektion und Tragen von Schutzhandschuhen - zu beachten.

Um einen hohen Patientendurchlauf gewährleisten zu können, wird oft die Vorgabe des BfArM nicht berücksichtigt. Statt nach jeder Kontrastmittelinjektion bzw. nach Verbrauch des Spritzenvolumens das gesamte System auszuwechseln, werden die Injektorspritzen wieder befüllt und somit für mehrere Patienten über einen längeren Zeitraum verwendet. Hierdurch sollen der Zeitverlust infolge von Aufbau und Befüllung sowie die Kosten - da z.T. erhebliche Kontrastmittelüberstände ungenutzt verworfen werden müssten - gesenkt werden.

In einer Studie konnte zwar gezeigt werden, dass durch eine korrekte Händehygiene das Kontaminationsrisiko im Rahmen des einmaligen Aufbaus und mehrmaliger Wiederbefüllung deutlich gesenkt werden kann, dass aber nach ca. 4-maligem Auffüllen Kontaminationen wieder nachweisbar sind [1-3]. Durch die Verwendung von Spezialeinwegventilen, sog. Rückschlagventilen, zwischen T-Schlauch und patientenseitigem Injektionsschlauch kann man entgegenwirken. Diese werden mit dem Patientenschlauch nach jeder Untersuchung gewechselt. Alternativ bieten Hersteller zur Einmalverwendung bereits mit Kontrastmittel zw. Kochsalzlösung vorgefüllte Injektorspritzen an.

Injektorspritzen sind gemäß BfArM nur für die Einmalverwendung zugelassen. Somit sind zeitlich versetzte Mehrfachentnahmen oder Wiederbefüllung zu unterlassen. Prinzipiell ist vor jedem Öffnen bzw. Anstechen einer Kontrastmittelflasche eine Händedesinfektion durchzuführen und anschließend sind Handschuhe zu tragen.

Neben der Beschriftung mit Anbruchdatum samt Uhrzeit bei Kontrastmitteln gehört auf folgende weitere Artikel die Datumsbeschriftung:

- Desinfektionsmittel

- Vernebler bei Sauerstoffanschlüssen

- Medikamente

Medikamente sollten regelmäßig auf ihr Haltbarkeitsdatum überprüft werden und bei bald anstehenden Ablauf dementsprechend markiert werden.

Immer Hände desinfizieren vor jedem Öffnen einer Kontrastmittelflasche. Tragen Sie anschließend Handschuhe.

\section{Organisatorische Maßnahmen}

Die Zuweiser sollten angehalten werden, bereits bei der Anmeldung von Untersuchungen Informationen zum Infektionsstatus des Patienten anzugeben. Im Rahmen der digitalen Anmeldung über KIS/RIS kann man dies sehr einfach einfordern, indem ein Pflichtfeld „Infektionsstatus und Erreger“ im System eingerichtet wird. Bei Nichtausfüllen dieses Kästchens kann somit aufgrund der Unvollständigkeit einer Anmeldung keine Freigabe dieser erfolgen. So kann bereits im Vorfeld geklärt werden, ob und in welcher Art Vorkehrungen zur Untersuchung eines infektiösen Patienten getroffen werden müssen, um das Risiko einer Raum- und Gerätekontamination ebenso wie Folgekosten aufgrund von Reinigung der Räumlichkeiten oder gar Raumsperrungen und damit verbundenen Untersuchungsausfällen zu reduzieren.

Des Weiteren sollten - wenn möglich - so wenig Materialien, Gegenstände oder Gerätschaften im Untersuchungsraum gelagert werden, um den Desinfektionsaufwand bei nachträglich erwiesenem Erregernachweis zu minimieren. Falls das Aufbewahren unumgänglich ist, muss bei den vorhandenen Schränken auf ein möglichst dichtes Verschließen von Türen bzw. Schubladen geachtet werden. Im Falle der Infektion bzw. Verdacht auf Infektion mit Tbc-Erregern oder Noroviren eines Patienten müssen alle beweglichen Gegenstände und Materialen aus dem Raum entfernt werden.

Ist der Infektionsstatus bei der Anmeldung bekannt, sollte entweder der zuständige Gerätearzt oder ein für den Bereich verantwortlicher Hygienebeauftragte oder auch Krankenhaushygieniker zur Beratung hinzugezogen werden, um über Art und Umfang der Hygienemaßnahmen sowie die Terminierung der Untersuchung zu entscheiden. Wenn möglich, sollten solche Untersuchungen am Ende des Arbeitstages in Abhängigkeit der Dringlichkeit stattfinden. Um eventuelle Raumkontaminationen und Infektionsgefährdung der Mitarbeiter zu minimieren, ist es sinnvoll, sich auf so wenig Räume und Mitarbeiter mit direktem Patientenkontakt (Aufklärung, Lagerung, Untersuchungsdurchführung) zu beschränken. Des Weiteren sollten gesonderte Wartezonen vorhanden sein.

Kenntnisse über mögliche Übertragungswege während des Aufenthaltszeitraums in einer radiologischen Abteilung auf das Personal und andere Patienten sind entscheidend, um den Schutz des genannten Personenkreises durch adäquate Hygienemaßnahmen gewährleisten zu können. Grundlegende hygienische Maßnahmen sorgen zwar für eine Unterbrechung des Übertragungsweges, müssen aber bei Vorlage des Erregernachweises um dessen spezifische Hygienemaßnah- 
Tab.4 Überblick über spezifische Hygienemaßnahmen.

\begin{tabular}{|c|c|c|c|}
\hline Erreger & Personal & Patient & Besonderheiten \\
\hline $\begin{array}{l}>\text { MRSA } \\
>\text { ORSA }\end{array}$ & $\begin{array}{l}\text { Schutzkittel } \\
\text { Handschuhe } \\
\text { Operationsmaske (Mund-Nasen- } \\
\text { Schutz) } \\
\text { Haarschutz }\end{array}$ & $\begin{array}{l}\text { 1) stationär: } \\
\text { Schutzkittel } \\
\text { - Handschuhe } \\
\text { Operationsmaske (Mund-Nasen- } \\
\text { Schutz) } \\
\text { Haarschutz } \\
\text { 2) ambulant: } \\
\text { Operationsmaske (Mund-Nasen- } \\
\text { Schutz) }\end{array}$ & $\begin{array}{l}\text { Untersuchung erst nach MRSA- } \\
\text { Screening } \\
\text { Aufenthalt des Patienten in } \\
\text { gesonderten Wartezonen }\end{array}$ \\
\hline $\begin{array}{l}3 \mathrm{MRGN} \\
4 \mathrm{MRGN}\end{array}$ & $\begin{array}{l}\text { Schutzkittel } \\
\text { Handschuhe } \\
\text { ggf. Operationsmaske (Mund-Nasen- } \\
\text { Schutz) }\end{array}$ & $\begin{array}{l}\text { Schutzkittel } \\
\text { Handschuhe } \\
\text { Operationsmaske (Mund-Nasen- } \\
\text { Schutz) }\end{array}$ & $\begin{array}{l}\text { Aufenthalt des Patienten in } \\
\text { gesonderten Wartezonen }\end{array}$ \\
\hline ESBL & $\begin{array}{l}\text { Schutzkittel } \\
\text { Handschuhe } \\
\text { ggf. Operationsmaske (Mund-Nasen- } \\
\text { Schutz) } \\
\text { - Haarschutz }\end{array}$ & $\begin{array}{l}\text { Schutzkittel } \\
\text { Handschuhe } \\
\text { Operationsmaske (Mund-Nasen- } \\
\text { Schutz) }\end{array}$ & $\begin{array}{l}\text { Aufenthalt des Patienten in } \\
\text { gesonderten Wartezonen }\end{array}$ \\
\hline VRE & $\begin{array}{l}\text { Schutzkittel } \\
\text { Handschuhe } \\
\text { ggf. Operationsmaske (Mund-Nasen- } \\
\text { Schutz) } \\
\text { - Haarschutz }\end{array}$ & $\begin{array}{l}\text { Schutzkittel } \\
\text { Handschuhe } \\
\text { Operationsmaske (Mund-Nasen- } \\
\text { Schutz) }\end{array}$ & $\begin{array}{l}\text { Aufenthalt des Patienten in } \\
\text { gesonderten Wartezonen }\end{array}$ \\
\hline Norovirus & $\begin{array}{l}\text { Schutzkittel } \\
\text { Handschuhe } \\
\text { Operationsmaske (Mund-Nasen- } \\
\text { Schutz) }\end{array}$ & $\begin{array}{l}\text { Schutzkittel } \\
\text { Handschuhe }\end{array}$ & bei Erbrechen FFP2-Maske \\
\hline $\begin{array}{l}\text { - Hepatitis B } \\
\text { - Hepatitis C }\end{array}$ & $\begin{array}{l}\text { Schutzkittel } \\
\text { Handschuhe }\end{array}$ & & $\begin{array}{l}\text { Schutzbrille bei Gefahr der Tröpfchen- } \\
\text { übertragung } \\
\text { mehrfach wiederholte Händedes- } \\
\text { infektion (verlängerte Einwirkzeit) }\end{array}$ \\
\hline HIV/AIDS & Handschuhe & & $\begin{array}{l}\text { Schutzbrille nur bei Gefahr der Tröpf- } \\
\text { chenübertragung }\end{array}$ \\
\hline $\begin{array}{l}\text { Tbc: } \\
\text { 1) pulmonal } \\
\text { offen } \\
\text { geschlossen } \\
\text { 2) extrapulmonal }\end{array}$ & $\begin{array}{l}\text { zu 1): } \\
\text { PFP2-Maske mit Exspirationsventil } \\
\text { Schutzkittel } \\
\text { > Handschuhe } \\
\text { - Haarschutz }\end{array}$ & $\begin{array}{l}\text { zu 1): } \\
\text { Operationsmaske (Mund-Nasen- } \\
\text { Schutz) oder besser } \\
\text { FFP2-Maske ohne Exspirationsventil }\end{array}$ & $\begin{array}{l}\text { zu 1): } \\
\text { Flächendesinfektion gem. } \S \S 17,18 \\
\text { IfSG (Scheuer-Wisch-Desinfektion) } \\
\text { durch entsprechend ausgebildetes } \\
\text { Personal (Desinfektor) }\end{array}$ \\
\hline
\end{tabular}

AIDS (engl.) - Aquired Immunodeficiency Syndrome; HIV (engl.) - Human Immunodeficiency Virus; 3MRGN - Multiresistente gramnegative Stäbchen mit Resistenz gegen 3 der 4 Antibiotikagruppen); 4MRGN - Multiresistente gramnegative Stäbchen mit Resistenz gegen $\mathbf{4}$ der 4 Antibiotikagruppen; MRSA - Methicillin-resistenter Staphyloccocus aureus; ORSA - Oxacillin-resistenter Staphyloccocus aureus; VRE - Vancomycin-resistente Enterokokken; ESBL - Extended-Spectrum-Betalaktamasen; TBC - Tuberculose

men erweitert werden ( $\bullet$ Tab.4). Hierbei gilt es zwischen direktem und indirektem Kontakt zu unterscheiden.

Kontaminierte Schutzkleidung sollte bereits im Untersuchungsraum abgelegt und in gesondert als Kontaminationsmüll gekennzeichneten Behältnissen entsorgt werden, um Eigenübertragung zu vermeiden. Diese Umverpackungen sollten sicher verschließbar sein. Für spitze und scharfkantige Gegenstände werden hierfür geeignete Behältnisse bereitgestellt. Die abschließende Händedesinfektion ist wie die abschließende Geräte- und eventuell Raumdesinfektion (Schlussdesinfektion) obligat. Hierbei ist es zwingend erforderlich die spezifischen Einwirkzeiten zugelassener Flächendesinfektionsmittel gem. VHA-Liste zu beachten.
Um eventuelle Raumkontaminationen und Infektionsgefährdung der Mitarbeiter zu minimieren, ist es sinnvoll, sich auf wenige Räume und Mitarbeiter mit direktem Patientenkontakt zu beschränken.

\section{Abstract}

$\nabla$

Radiology plays an important part in the hospital hygiene due to the huge number of examinations and daily patient contact through the staff. Therefore the hygiene education of the staff is mandatory. Additionally, it is very important to optimize the workflow and to name hygiene responsibilities such as radiographers and physicians. 
Kernaussagen

- Aufgrund eines sehr großen Durchlaufs mit fast ausschließlich direktem Kontakt zu Patienten nehmen radiologische Abteilungen auch in der Krankenhaushygiene eine zentrale Bedeutung ein.

- Um den Arbeitsablauf von radiologischen Abteilungen so wenig wie möglich zu stören, ist es wichtig, die Hygienebeauftragten in die Erstellung von Organisationsstrukturen durch den Krankenhaushygieniker einzubinden.

- Unangepasste Hygienemaßnahmen verlangsamen oder unterbrechen den Arbeitsablauf und spezifische Hygienemaßnahmen sind z.T. sehr kosten- und zeitintensiv.

- Daher ist es wichtig, alle Mitarbeiter einer solchen Abteilung regelmäßig zu Themen der Hygiene zu schulen, damit das Personal alle allgemeinen und spezifischen Hygienemaßnahmen sicher umsetzen kann.

\section{Literatur}

1 Buerke B, Sonntag AK, Fischbach R et al. Injektionsautomaten in der Magnetresonanz- und Computertomographie: Pilotstudie zu hygienischen Aspekten. Fortschr Röntgenstr 2004; 176: 1832 - 1836

2 Buerke B, Mellmann A, Stehling C et al. Microbiologic contamination of injectors at MDCT: experimentel and clinical investigations. Am J Roentgenol 2008; 191: W283-287

3 Buerke B, Puesken M, Mellmann A et al. Microbiologic contamination and time efficiency of automatic MDTC injectors with prefilledsyringes: Result of clinical investigation. Am J Roentgenol 2010; 194: 299 $-303$

4 Bundesgesundheitsblatt. Händehygiene. Gesundheitsforsch - Gesundheitsschutz. 2000; 43: 230-233

5 Deutsche Gesellschaft für Krankenhaushygiene (DGKH)Leitlinie: Hygienebeauftragte ${ }^{\circledR}$ in Pflegeeinrichtungen und anderen betreutenund gemeinschaftlichen Wohnformen - Anforderungen und Aufgaben. Im Internet: www.krankenhaushygiene.de (Stand 22.11.2012)

6 Gesetz zur Verhütung und Bekämpfung von Infektionskrankheiten bei Menschen (Infektionsschutzgesetz - IfSG). Im Internet: www.gesetze-im-internet. de (Ausfertigungsdatum: 20.7.2000)

7 Gesetz- und Verordnungsblatt (GV.NRW). Verordnung über Hygiene und Infektionsprävention in medizinischen Einrichtungen (HygMedVO). Im Internet: www.recht.nrw.de (Ausgabe 2012 Nr. 8 vom 30.3. 2012, Seite 139-154)

8 Kock R, Becker K, Cookson B et al. Methicillin-resistant Staphylococcus aureus (MRSA): burden of desease and control challenges in Europe. Euro Surveill 2010; 15: 19688

9 Robert Koch Institut. Infektionen/krankenhaushygiene/Desinfektion. Im Internet: www.rki.de (Stand 16.8.2014)

10 www.wikipedia.org (Stand 16.8.2014)

\section{Über die Autorin}

$\nabla$

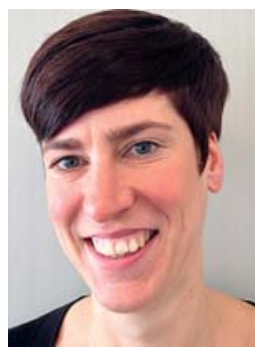

Annette Saur

Jahrgang 1973. 1993-1995

Ausbildung zur MTRA am Universitätsklinikum des Saarlandes, Homburg/Saar. 1995-2014 Tätigkeit als MTRA im Institut für Diagnostische und Interventionelle Radiologie am Marien Hospital Düsseldorf. Seit 2014 Ltd. MTRA am Institut für Diagnostische und Interventionelle Radiologie und Neuroradiologie, Universitätsklinikum Essen.

\section{Korrespondenzadresse}

Annette Saur, Ltd. MTRA

Institut für Diagnostische und

Interventionelle

Radiologie und Neuroradiologie

Universitätsklinikum Essen (AöR)

Hufelandstraße 55

45147 Essen

Deutschland

Tel. $+49(0) 201 / 723-8450$

Fax: +49(0)201/723-1850

E-Mail: Annette.Saur@uk-essen.de

Interessenkonflikt: kein Interessenkonflikt angegeben

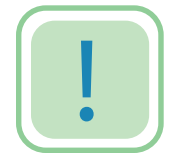

\section{Radiobonus}

Wer die CRTE-Fragen zu diesem Artikel bis zum 31.Januar 2015 beantwortet, kann eines von 3 Exemplaren des Werks „Kappstein: Nosokomiale Infektionen“ gewinnen.

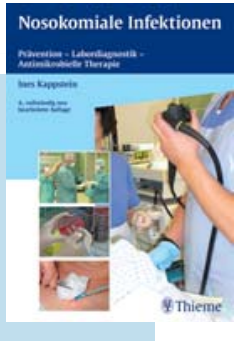




\section{CRTE-Fragen Radiologie - Das Drehkreuz in der Medizin: Hygiene in der Radiologie}

1 Nosokomiale Erkrankungen beruhen auf Infektionen, die

A. ausschließlich während eines stationären Aufenthaltes in Krankenhäusern oder Pflegeeinrichtungen erworben wurden

B. nur direkt übertragen werden

C. nur indirekt übertragen werden

D. ausschließlich Wunden besiedeln

E. die der Patient bereits vor einem stationären Aufenthalt erworben hat

\section{Welche Aussage ist nicht zutreffend?}

A. Hygienebeauftragte erstellen in Zusammenarbeit mit dem Krankenhaushygieniker Maßnahmenblätter speziell für die Abteilung

B. Hygienebeauftragte führen regelmäßig eine Begehung der Abteilung durch

C. Hygienebeauftragte sind ausschließlich für Schulungen der Mitarbeiter zuständig

D. Hygienebeauftragte überprüfen regelmäßig Arbeitsabläufe bezüglich Desinfektion, Reinigung und Entsorgung

E. Hygienebeauftragte sind verpflichtet, sich regelmäßig fortzubilden; spätestens alle 2 Jahre

\section{Wann führt man eine Händedesinfektion durch?}

A. vor und nach jedem Patientenkontakt

B. ausschließlich vor dem Anziehen der Schutzhandschuhe

C. nur nach dem Ablegen der Schutzhandschuhe

D. bevor ein Instrumententisch steril eingedeckt wird

E. ausschließlich nach mehrstündigem Tragen von Schutzhandschuhen

4 Im Umgang mit Flächendesinfektionsmitteln ist Folgendes wichtig:

A. Das Abtrocknen des Desinfektionsmittels auf Oberflächen als Einwirkzeit ist ausreichend

B. Die spezifische Einwirkzeit und Konzentration bei bestimmten Erregern sowie prinzipiell das vollständige Benetzen von Oberflächen zu beachten

C. Als Flächendesinfektionsmittel kann auch ein Händedesinfektionsmittel eingesetzt werden

D. Für die Flächendesinfektion ist es ausreichend, ein Produkt der VHA-Liste zu verwenden

E. Die dafür vorgesehenen Behältnisse werden immer vor den Untersuchungsräumen bereitgestellt

\section{5}

A. ersetzt eine Händedesinfektion vor Untersuchungsbeginn, wenn Schutzhandschuhe verwendet werden

B. ersetzt die Händedesinfektion nach dem Ablegen dieser

C. verringert den Verbrauch von Händedesinfektionsmitteln

D. erfordert dennoch eine Händedesinfektion vor Verwendung bzw. nach Ablegen der Schutzhandschuhe

E. ist nur wichtig bei Reinigungsarbeiten

\section{Welche Aussage trifft zu?}

A. Uhren und Schmuck an Handgelenken sowie Ringe müssen während der Arbeitszeit abgelegt werden

B. Der Haarschutz wird nur aufgesetzt, die Haare müssen nicht vollständig verdeckt sein

C. Der Mund-Nasen-Schutz kann während der Untersuchung zeitweilig abgenommen werden

D. Privatkleidung darf getragen werden, dabei ist zwar das Tragen eines Schutzkittels notwendig; dieser muss allerdings vorne nicht geschlossen werden

E. Ein Schuhwechsel macht eine Händedesinfektion nicht zwingend erforderlich

7 Welche Aussage ist richtig? Das Anstechen von einer z. B. Kontrastmittelflasche

A. sollte nur nach dementsprechender Händedesinfektion erfolgen

B. sollte erst nach entsprechender Händehygiene - Händedesinfektion und Tragen von Schutzhandschuhen erfolgen

C. kann mehrfach wiederholt werden, wenn nur kleine Mengen entnommen wurden

D. kann nur einmalig erfolgen und die gesamte Kontrastmittelmenge muss vollständig aufgebraucht werden unabhängig davon, ob zwischenzeitlich ein infektiöser Patient untersucht wurde

E. ist nur wichtig bei Reinigungsarbeiten

\section{Was sollte beim Umgang mit infektiösen Patienten} beachtet werden?

A. Schutzkleidung ist nicht erforderlich

B. Die Untersuchung sollte möglichst auf das Ende des Arbeitstags terminiert werden

C. Eine Oberflächendesinfektion ist nicht nötig

D. Der Infektionsstatus muss nicht bei der Anforderung vermerkt werden

E. Eine Untersuchung in der Radiologie ist nicht möglich 


\section{Was ist bei Tbc-infizierten Patienten zu beachten?}

A. Die Tbc-Infektion muss im Rahmen der Hygienemaßnahmen nicht näher differenziert werden

B. Der Erreger befällt ausschließlich die Lunge

C. Der Erreger ist nicht auf Dritte übertragbar, wenn die Kontaktperson dagegen geimpft ist

D. Tbc in der pulmonalen offenen Form macht das Tragen von FFP2-Masken für Patient und alle an der Behandlung Beteiligten notwendig

E. Eine besondere Form der Raumhygiene ist nicht erforderlich

\section{0}

A. werden Materialien streng getrennt zwischen Glasabfall, aus spitzen oder scharfkantigen Gegenständen bestehendem Abfall, Restabfall sowie Kontaminationsabfall

B. sollte das Behältnis für mit Erregern kontaminierte Materialien bereits im Untersuchungsraum aufstellt werden

C. Oberflächen und Gerätschaften sollten mehrmals täglich sowie nach jedem Patientenkontakt desinfiziert werden

D. Schutzhandschuhe - unsteril und steril - können mikroskopisch kleine Löcher aufweisen können. Daraus ergibt sich die prinzipielle Händedesinfektion vor und nach dem Tragen von Schutzhandschuhen

E. werden die dafür vorgesehenen Behältnisse immer vor den Untersuchungsräumen bereitgestellt 